\title{
Exploring Quantitative and Qualitative Methodologies: A Guide to Novice Nursing Researchers
}

\author{
Jordan Tovera Salvador, PhD, RN \\ University of Dammam, Kingdom of Saudi Arabia
}

doi: 10.19044/esj.2016.v12n18p107 URL:http://dx.doi.org/10.19044/esj.2016.v12n18p107

\begin{abstract}
This paper reviews three research articles concerning the methodologies of quantitative and qualitative research. The body of knowledge can be used as a guide for novice researchers who wanted to have in-depth understanding about the nature and underpinnings of each research design. The first article written by Beverly Hancock, 'Trent for Research and Development in Primary Care: An Introduction to Qualitative Research (2002)', emphases on the foundations of qualitative research in application to primary health care setting; the second article authored by Gary Rolfe, 'Validity, Trustworthiness, and Rigor: Quality and the Idea of Qualitative Research' (2006), accentuates the methodological 'issues in nursing research; and the last article written by Looi Theam Choy, 'The Strengths and Weaknesses of Research Methodology: Comparison and Complimentary between Qualitative and Quantitative Approaches' (2014), spotlights the similarities and differences of both research methodologies in the field of social sciences. Fundamentally, it would specify a necessary knowledge, adequate information, and appropriateness in the applicability of the research design. Likewise, it would stipulate a realization for the readers about the coexistence of both research approaches in any field of investigation.
\end{abstract}

Keywords: Nursing Research, Qualitative Research, Quantitative Research, Research Methodology

\section{Introduction}

This paper recapitulates three articles concerning the various selections of research methodologies of quantitative and qualitative research. The first article emphases on the foundations of qualitative research in application to primary health care setting; the second article accentuates the methodological issues in nursing research; and the last article spotlights the 
similiarities and differences of quantitative and qualitative research methodologies in the field of social sciences.

This articles's objectives can be summarized as follows:

1. Define essential terms related to the articles that may shed light and give awareness to the readers (remember).

2. Abridge the articles the way it would be certainly comprehended (understand).

3. Outline the diverse qualitative research designs, nature, and applications (apply).

4. Compare and contrast the methodologies issues in quantitative and qualitative research (analyze).

5. Convoke reaction in terms of new learning, application, and challenges (evaluate).

6. Concoct a conclusion for the three (3) articles (create).

In addition, this paper conveys insights and discussions relating to distinctive outlook of qualitative and quantitative research predominantly on the selected articles focusing much with the philosophical underpinnings of qualitative research design and methodology. Fundamentally, it would specify a necessary knowledge, adequate information, and appropriateness in the applicability of the research design in the chosen field of investigation. Besides, it would give the reader comprehensive understanding of the essential criteria of qualitative research: validity, trustworthiness, and rigor; which are all major methodological issues in qualitative nursing research. Furthermore, it would stipulate a realization for the readers about the coexistence of both research approaches in any field of investigation.

\section{Introduction: Quantitative Versus Qualitative Research}

Over the centuries, research designs had been subjected for numerous debates concerning its aspects, specifically with its purposes, approaches, methodologies, independence, and samples. Various researchers and authors had previously drawn the borderlines amid the two designs like the fact that qualitative research is an inductive method of reconnoitering the experiences of human beings towards social phenomena to discover the essence of such occurrences (Creswell, 2013. p. 4) and quantitative research is a 'deductivist, objectivist, and positivist' method of research that involves numbers and quantification in collecting and analyzing data (Bryman, 2012. p. 715). Nevertheless, due to the new emerging paradigms (Bryman, 2008) and further evaluations of the strengths and weaknesses of both designs. Choy (2014) determined that 'there is no perfect between qualitative and quantitative research methodologies'. Therefore, a comparison of results for both research approaches can be a way of elucidating its 'limitations and biases' (p. 104). Hence, this is the intention why mixed methods research 
came into the picture, 'to integrate qualitative and quantitative research within a single project' (Bryman, 2012. p. 628).

The table below displays the differences of qualitative and quantitative designs in reference to its corresponding research aspects identified by Bryman (2012, p. 36), Creswell (2013, p. 18), and Polit and Beck (2011, p. 13):

\begin{tabular}{|c|c|c|}
\hline Quantitative Research & Aspect & Qualitative Research \\
\hline Deductive (Testing of Theory) & Theory & Inductive (Generating Theory) \\
\hline $\begin{array}{c}\text { Positivism (Natural Science } \\
\text { Model) }\end{array}$ & Epistemological Orientation & Interpretevism \\
\hline Objectivism & Ontological Orientation & Constructionism \\
\hline $\begin{array}{l}\text { Values and Biases are to be held } \\
\text { in Check; Objectivity is Sought }\end{array}$ & Axiological Orientation & $\begin{array}{l}\text { Subjectivity and values are } \\
\text { Inevitable and Desirable }\end{array}$ \\
\hline $\begin{array}{c}\text { Post-positivist Knowledge } \\
\text { Claims }\end{array}$ & Philosophical Assumptions & $\begin{array}{c}\text { Transformational Knowledge } \\
\text { Claims }\end{array}$ \\
\hline Survey and Experiments & Strategies of Inquiry & $\begin{array}{c}\text { Narratives, Case Study, } \\
\text { Phenomenology, Grounded } \\
\text { Theory, and Ethnography, Case }\end{array}$ \\
\hline $\begin{array}{c}\text { Fixed Approaches, Numerical } \\
\text { Data, and Close-Ended } \\
\text { Questions }\end{array}$ & Employment of Methods & $\begin{array}{l}\text { Emerging Approaches, Text/ } \\
\text { Image Data, and Open-Ended } \\
\text { Questions, }\end{array}$ \\
\hline $\begin{array}{c}\text { Test Theories/Explanations } \\
\text { Ascertains Variables } \\
\text { Hypotheses } \\
\text { Validity and Reliability } \\
\text { Numerical Measures } \\
\text { Unbiased Approaches } \\
\text { Statistical Treatment } \\
\text { Focus on the Product } \\
\text { Seeks Generalizations } \\
\text { Large and Representative } \\
\text { Samples } \\
\text { Fixed and Pre-specified Design } \\
\text { Tight Control over Context }\end{array}$ & Methodological Orientations & $\begin{array}{c}\text { Gathers Participant Meanings } \\
\text { Single Concept/Phenomenon } \\
\text { Inclusion of Personal Values } \\
\text { and Understands the Setting of } \\
\text { Participants } \\
\text { Validates Finding and Interprets } \\
\text { Data } \\
\text { Has Reform Agenda } \\
\text { Collaboration with the } \\
\text { Participants } \\
\text { Focus on the Process and } \\
\text { Product } \\
\text { Seeks In-Depth Understanding } \\
\text { Small Informational-Rich } \\
\text { Samples } \\
\text { Context-Bound, Flexible, and } \\
\text { Emergent Design }\end{array}$ \\
\hline
\end{tabular}

Table 1.1 Differences of Qualitative and Quantitative Research According to Bryman (2012, p. 36), Creswell (2013, p. 18), and Polit and Beck (2011, p. 13):

\section{Trent Focus for Research and Development in Primary Health Care: An Introduction to Qualitative Research}

This article was termed by the author, Beverly Hancock (2002) as a 'resource pack' for professionals in the primary health care setting to learn the theoretical and foundational underpinnings of qualitative research (p. 1). It is indispensable to identify the methodology of this research design in order to delve with potential research developments, which interests social experiences and problems. Likewise, this starter pack would introduce improvement in understanding qualitative research. 
The resource pack was divided into six (6) sections: (1) nature, (2) design, (3) data collection, (4) handling data, (5) analyzing results, and (6) presentation of results. All of the sections briefly explained the theoretical underpinnings of qualitative research with some exercises to gauge the readers' knowledge and understanding about the given topics.

The first two (2) sections tackled the philosophical underpinnings and diverse designs of qualitative research. Hancock (2002) momentarily expounded the nature and proponents of qualitative research design with the subsequent significant details:

- $\quad$ The foremost emphasis is on the understanding of social phenomena.

- $\quad$ Ask queries about human behavior, formations of opinions and attitudes, cultural perspectives, affectation towards specific phenomenon, and variation among clusters of ethnic or group of people in the society.

- $\quad$ Questions are open-ended, which begins with 'how and why'.

- Holistic approach is the manner of understanding in qualitative research.

- Interviews, observations, and utilization of audio-visual recording are some of the research tools used in gathering subjective data from the participants.

- $\quad$ Essence of trustworthiness in qualitative research.

- Inductive approach in developing concepts and theories about understanding human experience.

Qualitative research has numerous popular research designs that are extensively used all over the world: phenomenology, ethnography, grounded theory, and case study. Each of this design has its own theoretical and philosophical underpinnings that researchers must understand to effectively come up with an excellent qualitative study. Phenomenology is the study of phenomena. Various authors offered several definitions of phenomenology including the following: 'focuses on the lived experiences of humans and is an approach to learning what the life experiences of people are like and what they remain' (Polit and Beck, 2011. p. 69), 'a phenomenon's essence and essentials that determine what it is' (Saldana, 2011. p. 7), 'study of people's perception of the world (as opposed to trying to learn what 'really is' in the word' (Willis, 2007. p. 107), 'a philosophy and research method designed to explore and understand people's everyday lived experiences ‘ (Abu Shosha, 2012. p. 31) and 'science of examples' (Van Manen, 1989. p. 26). All of these descriptions are recognized and used as references for phenomenological research. The author mentioned that phenomenology do not provide the detailed explanation towards the phenomena, however, it postulates essences and insights for people to better understand the phenomena occurring in the social world (Hancock, 2002. p. 4). 
Ethnography is an anthropological descriptive study of the culture of certain ethnic groups. Hancock (2002), mentioned four (4) cultural parameters for ethnography: 'geographical (particular region or country, religious, tribal, and shared experiences' (p. 5). Willis (2007), denoted ethnography and fieldwork as the same concept on his book that means 'learning about the culture through interviewing and gathering data in an authentic environment'. Piloting this study would take a lot of time (Creswell, 2013. p. 14) including the ocular visit, immersion, fieldwork, and follow-up.

Grounded Theory yields 'new knowledge' that can be developed into a theory about a phenomenon (Hancock, 2002, p. 5). Bryman (2012) modified this definition as 'derived from data, systematically gathered analyzed through the research process' (p. 387). Just like other research designs, interviews, observations, and documents are used to produce data for extensive data analysis.

Case study can be both qualitative and quantitative (Hancock, 2002. p. 6). Case study is an intensive exploration of a specific subject (Bryman, 2012. p. 709). The more cases are included in the study the more complex the analysis would be. Case studies offer a lot of avenues for prospective research topics in the primary health care settings. However, stipulation about case study had been subjected to a lot of debates regarding to its credibility and reliability because a case does not represent the whole population. Experts clearly stated that case studies are particularistic and contextual. The correlation of the case will be indentified by the researcher from the population of the research locale. (Hancock, 2002. p. 7).

The last four (4) sections were all about the research methodology of qualitative research. In spite of the different philosophical underpinnings of this research design, it only circumnavigate with numerous methods of collecting and analyzing qualitative data. Instrumentation for qualitative research design includes observations, individual dialogues/interviews, focus group discussions, field notes, audio-visual recordings, and use of qualitative documents, and photographs/artifacts. Conversely, ways of collecting qualitative data should be ethically accepted and implemented. Informed consent from the participants should be facilitated, because in any kind of research 'there is always some potential for harm' (Hammersley \& Traianou, 2012. p. 74). As soon as data were collected either using one of the methods, handling qualitative research data is done through transcribing the qualitative data from the recorded device (audio or visual). Transcribing data is a timeconsuming activity. Presently, tape analysis (getting information by repeating playbacks) is already used, however, this method may produce biases from novice researchers. 
Analyzing qualitative data has two (2) essential steps: coding and content analysis. Coding as defined by Bryman (2012), 'process whereby data are broken down into component parts, which are given names' (p. 710) and Polit and Beck (2011), 'process of transforming raw data into standardized form for data processing, analysis, and in the process of identifying and indexing recurring words, themes, or concepts within the date' (p. 722). Coding may differ from every researcher. Some uses letters (a, b, c, d, e), numbers $(1,2,3,4,5)$ and others uses color coding scheme (highlighting in different colors). Content analysis is a method of 'categorizing the qualitative data according to classification, summarization, and tabulation' (Hancock, 2002, p. 17). Moreover, content analysis may also vary according to already proven systematical steps from various proponents of qualitative studies like Giorgi, Van Kaam, Van Manen, Parse, and Collaizi. The usual steps are as follows: reading and re-reading of texts, extracting narrative statements, linking narratives to form categories, sorting out minor and major categories, reviewing of contextual categories, and assigning of sub-themes and themes. Nowadays, it is now easier to transcribe and analyze data through the use of electronic software packages like ATLAS, NVivo, and NUD*IST. The only problem with these advanced mechanisms is that it has language specifications, which local dialects could not be processed for transcription and data analysis.

The last section of the resource pack is the presentation of results of qualitative research. Results may be presented in the cluster of themes and contextual categories. Themes as defined by Polit and Beck (2011), as ' $a$ recurring regularity emerging from an analysis of qualitative data' (p. 744). Themes may be in form of 'speak for themselves' (Hancock, 2002. p. 23) or quantitatively.

The idea of this resource pack is to educate and establish a foundational knowledge for primary health care professionals to understand philosophical underpinnings of qualitative research. It gives an outline what should be expected for qualitative studies. It also briefly presented the various research designs applicable with primary health acre settings such as phenomenology (lived experiences of human being), ethnography (culture and people), grounded theory (formulate a theory based from phenomena), and case study (single in-depth study). Likewise, it discussed the research methodology and offered diverse research methods that researchers can employ (interviews, focus group discussions, and observation). Finally, it showcased the handling, analyzing, and presenting the qualitative data. Therefore, this resource pack is unquestionably suitable in educating professionals who would like to delve with qualitative research. 


\section{Validity, Trustworthiness, and Rigor: Quality and the Idea of Qualitative Research}

Qualitative research has faced so many challenges in the past half century. It had been the subject for corroboration among various researchers and scholars for the following reasons: 'too subjective and impressionistic, difficult to replicate, problems of generalization because the scope of the finding were restricted, and lack of transparency or how the author came up with such conclusions' (Bryman, 2012. p. 405-406). All of these were being questioned for its validity, authenticity, transferability, and credibility. The questions researchers commonly asked about were: does qualitative research have standard criteria to follow? Or 'Would it be useful to develop quality criteria into checklists?’ (p. 413). Sandelowski and Barroso (2002) begun to noticed these emerging problems in qualitative research considering they have already done resolving all the possible pitfalls they have identified in studying this design (p. 2). In the article written by Rolfe (2006), he stated that the main reason why qualitative research does not have any standard criteria to follow was because the nature and philosophical underpinnings of the research design has always been questionable over the time being ( $\mathrm{p}$. 305). The main challenge was - to make a consensus on how to evaluate the quality in qualitative research. How can this issue be resolved if various positions had been postulated over the years kept questioning the latest criteria in scrutinizing the quality in qualitative research?

Three (3) positions were recognized concerning this qualitative research insurgencies as stated by Rolfe (2006): (1) authors who desired to use the same criteria used in quantitative research, (2) authors who wanted to develop dissimilar criteria used in quantitative research, and (3) authors that questioned the 'appropriateness of the pre-determined criteria in determining quality in qualitative research' (p. 304).

The first position gave birth to rigor as one of the criterion for assessing and evaluating quality in qualitative research. Authors believed that reliability and validity in the quantitative perspectives could also be used in verifying the credibility of qualitative research. Morse et al. (2002) believed that when research processes have undergone rigorous manner, quality would be achieved (p. 13). Likewise, authors suppose that rigor lies in the capabilities of the researchers rather than the people reading the research.

Contrary to the first position, various authors disagreed the postulation of Morse et al. (2002) believe that some of the terminologies used to assess the quality of qualitative research borrowed from the quantitative were erroneous and not necessary for qualitative (Rolfe, 2006. p. 305). Instead of using 'validity' Sandelowski (1993) propped-up to use 'trustworthiness' for which he supposed to be more 'auditable' (p. 2). 
Consequently, trustworthiness had been already one criterion in assessing and evaluating the quality in qualitative research. Polit and Beck (2012) defined it as 'the degree of confidence qualitative researchers have in their data, assessed using the criteria of credibility, transferability, dependability, confirmability, and authenticity’ (p. 745). Identical postulations were drawn by Guba \& Lincoln (1989) and Graneheim \& Lundman (2004) about aligning the counterparts of trustworthiness to quantitative approach dividing it to sub-parts: transferability for external validity, dependability for reliability, credibility for internal validity, and confirmability. Nevertheless, Sandelowski was not convinced that reliability should not be used as a criterion for quality in qualitative research because of its element of repeatability to get a valid consensus in the data analysis, which is the focal purpose of the validity. So, she precluded reliability and focus on validity and trustworthiness (Rolfe, 2006. p. 305). After these endless discussion concerning the criteria for quality in qualitative research, authors have amalgamated all emerging positions and came up with three (3) criteria: rigor, trustworthiness, and validity.

Dialogues on the applicability of the criteria to qualitative studies had not concluded after its determination. Several disputes and rebuts were raised depending on the design researcher used. Criteria were not always germane with all kinds of research designs. This means that there are no definite criteria for qualitative research alone. Positivist approach will always be extant as methodology in the qualitative studies and vice versa. All these conflicting paradigms had been huge tussles in the world of nursing research. As long as there is an emerging paradigm on top of the other, another incongruity will be discovered and unceasing arguments will prompt to existence. Adding up to these bewilderments, were those authors like Morse and Field (1996) who published a textbook about qualitative research without 'clear-cut distinctions' between terms like 'qualitative approaches, perspective, paradigm, research, inquiry, findings, theory, researcher, and data'; Holloway and Wheeler (1996) and Munhall (2001) who made a merit between the nature of both research designs encompassing the terms like 'holistic approach, emic, contextualized, interpretive, immersed, humane experience, and descriptive/narrative data; and Streubert and Carpenter (1999) which created a 'list of characteristics of qualitative research' (Rolfe, 2006. p. 306-307).

The author would like to give emphasis that in spite of the efforts authors had put on discovering the appropriate standards for evaluation in qualitative research, the more failure they get into. Qualitative and quantitative research methodologies/methods will always be part of each other, no matter how many restrictions may researcher put into it. Rolfe (2006) concluded the following: 
- $\quad$ 'Whilst the term 'qualitative research' might be used accurately to describe methods of the data collection, it cannot adequately encompass the full range and diversity of non-quantitative methodologies.

- It is counterproductive to continue to regard al the qualitative research methodologies under a single 'interpretivist' or 'constructivist' paradigm.

- $\quad$ The search for a generic framework for assessing the quality of qualitative research should be abandoned in favor of individual judgments' of individual studies.' (p. 309).

Likewise, Rolfe (2006), suggested the use of 'detailed reflexive research diary' for both quantitative and qualitative research to have laidback audit trails of the research processes and methodologies as well as 'selfappraisal' and 'ongoing self-critique' of the researcher to give their readers the whole awareness of the research and concept (p. 309).

\section{The Strengths and Weaknesses of Research Methodology: Comparison and Complimentary between Qualitative and Quantitative Approaches}

The article written by Looi Theam Choy (2014) is a comparative and complimentary analysis of the strengths and weaknesses of the methodologies for both research designs through the use of secondary resources in the field of social sciences (p. 99). Fundamentally, methodology for quantitative research concerns to the measurement of the data either by surveys or experiments that will be applied with statistical treatment (Creswell, 2013. p. 155); on the other hand, qualitative research relates to texts or images data that has a 'unique data analysis and draw on diverse designs' (Creswell, 2013. p. 183). These rudimentary information are all known by a lot of people whether they have in-depth knowledge about research or a little bit of information about the methodology of a particular research design. This is the reason why researcher must distinguish the nature of research methodologies to prevent disastrous result of the study.

Somekh \& Lewin (2012) defined methodology as 'a collection of methods in which a particular piece of research is undertaken and judged to be valid' (p. 325). Other definition includes, 'methodology is the strategy or plan of action lies behind the choice and use of particular methods' (Crotty, 1998. p. 3, Scotland, 2012. p. 9). From the aforesaid definitions, methodology is the principal outline of what methods researchers' will be using for their studies regardless of the design based from their elected research paradigm. In fact, Guba \& Lincoln (1994) mentioned that methodology is one major component of research paradigms. Methodological questions dictate 'how can the inquirer (would-be-knower) go about finding out whatever he or she believes can be known' (p. 108). Methodology varies from dissimilar contending paradigms of research. At 
present, there are numerous methods for both qualitative and quantitative research designs, nevertheless, it corresponds to the philosophical underpinnings of the preferred paradigm what methods can be applied or not for the study. Research design and methods are often used interchangeably. Just like the example provided by Bryman (2012), 'the two terms are often confused... case study - is very often referred to as method. Case study entails the detailed exploration of a specific case, which could be a community, organization, or a person' (pg. 45). Simply, research design is a framework for the gathering and analyzing of data and research design is a technique for gathering the data such as survey questionnaires, semistructured interviews, experimentations, and observations.

Choy (2014) built comprehensive justifications on how qualitative and quantitative approaches in research methodologies were prepared. From the selection of topics, deciding the appropriate research design, setting scopes and delimitations of the study, gathering of review of related literature and studies, gathering the objective (quantitative) and subjective (qualitative) data, analyzing the data (statistical treatment/unique data explication), interpreting the data, and finally, reporting the results to other people.

As cited earlier, he intended to make a comparison of the two research designs or perhaps there would emerge an approving methodology that would resolve the ambiguities and downsides of both research designs and will yield enhance aftermaths. The results of Choy's investigations were presented in figure 1.1.

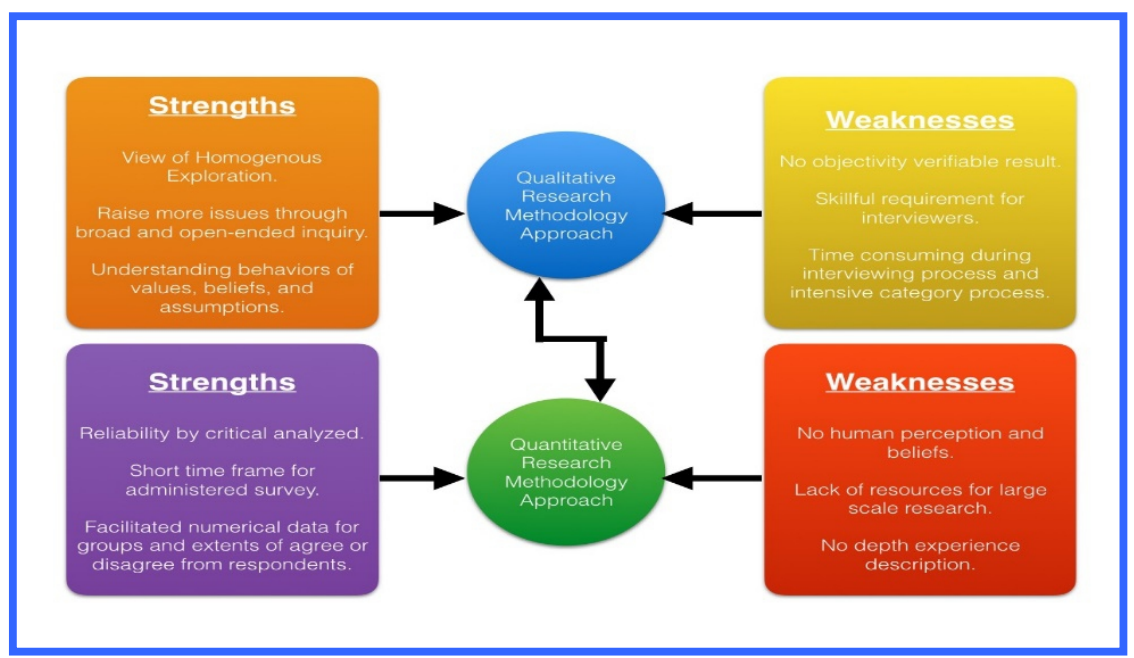

Figure 1.1 Model of Strength and Weaknesses of Qualitative and Quantitative Research Methodologies (Choy, 2014. p. 101). 
Figuring-out the strengths and weaknesses of both approaches from the review of related literature and to find-out its suitability, application was obtained by the investigator through secondary sources like journals, textbooks, books, online articles, and other relatable documents that can help discover the answer to this inquiry in the dearth of the primary resources (case study and content/ context analysis). Results and findings of the scrutiny divulge that the strengths and weaknesses revealed in the model of Choy were all correct. He supposed that both research methodologies from varied cases have good and weak arguments that need to be addressed by questionnaires for quantitative research that should be enhanced with openended questions and qualitative research should be augmented with survey since it has no dominance to randomize and no capability to control independent variables, thus, making higher chances of commiting erroneous interpretation. He assumed that weaknesses of the first design were unerringly the strengths of the latter and vice versa. For this purpose, Choy (2014) clinched that none of the two (2) research methodologies is impeccable rather the shortcomings of each research methodologies were the potencies to resolve the ambiguities of each methodology. Hence, he recommended that the identified similarities and differences should used as references for both independent methods of the same study to patch the prejudices and limitations identified.

\section{Reaction to New Learning, Application, and Challenges}

The articles cited above answered the perplexities of both research designs pertaining its philosophical keystones, research methodologies, and other trifling specifics.. There are a lot of things to refurbish as a novice researcher walkthrough the entire process of research whether quantitative or qualitative design.

The first article revisited the run-of-the-mill knowledge about qualitative research design. This is a great avenue for novice researchers to review the philosophical underpinnings of the distinctive qualitative research designs such as phenomenology (lived experiences), grounded theory (formulation of new theory from phenomena), case study (in-depth holistic study of individual cases), and ethnography (understanding culture of certain ethnic groups). Individual interviews, focus group discussions, and observation (audio-visual recording, photos, field notes, and artifacts) are amongst the most extensively used methods in collecting qualitative data. Equally, narratives form the participants of the study should be excellently transcribed (transcript file), even though, it would take too much of your time. Hancock (2002) mentioned in her research pack that it is not indispensible to transcribe all interviews and tape analysis would transcribe it easier (p. 14). Conversely, exhausting this technique upsurges the 
probabilities of the data collection to be curtailed to the extent of not reaching the participants' point of saturation, thus, interpretation eventually would turnout to be bias. Every researcher should keep in mind that transcripts should not denote any participants' names; pseudo names are commonly used to keep the anonymity of the participants. Furthermore, content analysis is essential in explicating the qualitative data. It clinches coding, labeling of data, which is a systematic manner of clustering and classifying the data either by number, letter, and color-coding schemes. Works of Giorgi, van Kaam, van Manen, and Collaizi were the most widespread data analysis methods used by researchers to arrive with the output of every qualitative study - themes. Since data are interpretative, subjective, copious, and holistic, it would be arduous to initiate the data analysis. So, structuring and classifying the data in minor and major categories would make it easier for a researcher to arrive with presenting the data into themes, which are 'recurring regularity emerging' from the data analysis (Polit and Beck, 2011. p. 744).

Applications of qualitative research in the practice of nursing would be advantageous not only for the nurses but for everyone. Rummaging into phenomenology as mentioned by van Manen (1989), 'writing distances from the life world, yet it also draws us more closely to the life world' (p. 29), researchers must appreciate the experiences of other people in lenses. Phenomenology is reconnoitering what is like to be in their shoes, thus, offering us insights in the true essence of the phenomena. In case study and ethnography, a holistic outlook of caring and understanding to an individual and even social group with distinct cultures and traditions a researcher can gain from the study. Nursing profession holds no boundaries when it comes to human caring science. Finally, grounded theory would introduce new breakthrough of theories that might be the key in refining the healthcare system in our world today (Saldana, 2011. p. 7).

The second article accentuates the methodological issues in nursing research. Contrasting quantitative study, results are reliable and undergone systematic and objective critical processes and experimentations before propping up with the results. It is no doubt proven by scientific inquiry. Nonetheless, qualitative data are still subjective. Interpretations may differ from one person to another, causing the validity and trustworthiness to be questionable. If validity and trustworthiness are questionable then rigor will be likewise be questioned too. This may imply that researcher manipulated the data and did not follow qualitative processes due to dubious and doubtful results. These were the reasons why Rolfe (2006) believed that the main reason why qualitative research does not have any customary criteria to follow because the nature and philosophical underpinnings of the research design has always been questionable over the time being (p. 305). I concur 
Rolfe's position that the preeminent way to resolve these issues is to construct a 'detailed reflexive research diary' for both quantitative and qualitative research to have an easy audit trail of the research processes and methodologies as well as 'self-appraisal' and ongoing self-critique' of the researcher to give their readers the whole idea of the research and concept (p. 309).

Finally, the last article spotlights the differences of both research design in the field of social sciences. Contemplating at the figure, it was evident that both research methodologies have their corresponding strengths and weaknesses that can generate advantages and disadvantages for the study. Quantitative methodology presented objective and reliable results, which can be dispensed in a short time frame and easily done through survey questionnaires, likewise, can be conveyed through numerical forms. Qualitative methodology surfaced exploration of homogenous views from the participants, opens a lot of opportunities for broad discussion questioning and probing, and the ability to understand the attitude, behavior, culture, and values of an individual or group of people. Both research methodologies have poles apart strengths. The strengths of the primary are the weaknesses of the latter and vice versa. Although Bryman (2012), stated in his book that even though both methodologies have different strengths and weaknesses, there are some correspondences both of the methodologies have in common in reducing and analyzing the data, essence of transparency, and both answers particular research questions. Both have pitfalls to resolve as mentioned by Choy, that comparison and complimentary results of both studies with the same research topics can answer the drawbacks of both research designs. On the other hand, adding up to the suggestions and recommendations of Choy, using mixed method research approach could help in lessening these dilemmas. Creswell (2013) pointed out in his table of comparison for quantitative, qualitative, and mixed methods that the latter could have both prearranged and merging methods. Data can be drawn in all possibilities and multiple forms, can use both statistical and text analysis, and can be across databases interpretations (p. 17). Furthermore, mixed method offers four (4) specific designs: (1) triangulation (Qual + Quant); (2) embedded (Qual(Quan)) or (Quan(Qual)); (3) explanatory (Quan supporting Qual); and (4) exploratory (Qual supporting Quan). Mixed method is deliberated as being 'cross sectional' (Polit \& Beck, 2011. p. 613) since it acquires the paramount of both worlds, qualitative and quantitative. Accordingly, it might be a possibility to cogitate in extenuating the difficulties of both methodologies. However, one more challenge of a researcher is to be accustomed and well known with the ideologies and principles of both approaches before engaging with mixed method research design. 


\section{Conclusion}

Understanding the fundamentals of research is amusing. Most of the time it stretches-out neurons for critical and systematic thinking. The abovementioned articles discoursed the philosophical underpinnings of qualitative research design and several indispensable foundations of quantitative research to compare and contrast. Thus, it aimed to give better understanding on how these variations can be resolved for enhance outcomes.

Since it is a fact, that most of the researchers, teachers, and students have wide-varieties of knowledge about quantitative research design; most of them perceive qualitative research design as a gray area. For others, they have no information about it at all. Qualitative research is promptly an emerging trend that most of the medical allied professions are instantly gearing at - from primary health care to various global systems of healthcare services. Researcher today would like to probe on the lived experiences of human beings towards specific phenomena (phenomenology), understanding cultural diversities from individual to societal perspectives (ethnography), discovering holistic approach from an comprehensive investigation of a single subject (case study), and discovering theories that can be used to improve perceptions and quality of health care system (grounded theory). Thus, qualitative research is a breath of fresh air for nursing research because it uncovers what quantitative results do not offer.

Knowing better the qualitative as a design, its methodology and methods would give the researcher the sense of producing a paper that meets the quality of a qualitative research: rigor, validity, and trustworthiness; which had been subjected to a lot of reflections up to the present day. Regardless of these scums, the insights, discernments, and supplementary learning from the aftermath of the research would matter most in the end.

As Choy (2014) concluded, both research designs have no perfect methodlogies (p. 104). The positive and negative elements of each research design can turn out to be opposite to each other. This is why comparative and complimentary results of same research topics may be valuable in lessening the acknowledged glitches of each research design. Today, researchers are inclined in considering mixed method research design as a choice to capture both upheavals of qualitative and quantitative research. Moreover, regardless of the design a researcher would like to inquire with as long as the methodology is in accordance with the design, methods are properly implemented, data are gathered with ethical considerations, data are suitably analyzed and employed with apposite statistical treatments, and on point conclusion and recommendations are postulated; then it is unquestionable that the research is absolutely on the right track. 


\section{References:}

Abu Shosha, G. (2012). Employment of Collaizzi's Strategy in Descriptive Phenomenology: A Refelction of a Researcher, European Scientific Journal, Vol. 8, No. 27, p. 31-43. ISSN:1857-7881 (Print); e-ISSN 1857-7431.

Bryman, A. (2008). The End of the Paradigm Wars?, in P. Alasuutari, J. Brannen, and L. Bickman (eds.), Handbook of Social Research. London: Sage.

Bryman, A. (2012). Social Research Methods (4 ${ }^{\text {th }}$ ed.) New York: Oxford University Press.

Choy, L. T. (2014). The Strengths and Weaknesses of Research Methodology: Comparison and Complimentary between Qualitative and Quantitative Approaches. IOSR Journal of Humanities and Social Sciences (IOSR-JHSS) (Vol. 19, Issue 4, Ver. III). E-ISSN: 2779-0837, P-ISSN: 2779-0845.

Creswell, J. W. (2009). Research Design: Qualitative and Mixed Methods Approaches. London: SAGE.

Crotty, M. (1989). The Foundations of Social Research. London: SAGE.

Denzin, N. K.; Lincoln, Y. S., eds. (2005). The Sage Handbook of Qualitative Research (3rd ed.). Thousand Oaks, CA: Sage. ISBN 0-76192757-3.

Given, L. M. (2008). The Sage encyclopedia of qualitative research methods. Los Angeles, California: Sage Publications. ISBN 1-4129-4163-6.

Graneheim, U. \& Lundman, B. (2004). Qualitative Content Analysis in Nursing Research: Concepts, Procedires, and Measures to Achieve Trustworthiness. Nurse Education Today 24, 105-112.

Guba, E. G. \& Lincoln, Y. S. (1989). Fourth Generation Evaluation. Sage, Newbury Park.

Guba, E. G. \& Lincoln, Y. S. (1994). Competing Paradigms in Qualitative Research. In N. K. Denzin, \& Y. S. Lincoln (eds.), Handbook of Qualitative Research (pp. 105-117) London: SAGE.

Hammersley, M. \& Traianou, A. (2012). Ethics in Qualitative Research: Controversies and Contexts. New Delhi, India: Sage Publication, Ltd.

Hancock, B (2002). Trent Focus for Research and Development in Primary Health Care: An Introduction to Qualitative Research. Trent Focus Group. University of Nottingham, Division of General Practice.

Hollaway, I. \& Wheeler, S. (1996). Qualitative Research for Nurses. Blackwell Science, Oxford.

Morse, J. M. (1991). Qualitative Nursing Research: A Free-for-all? In Qualitative Nursing Research: A Contemporary Dialogue (Morse, J., ed.), Sage, Newbury, pp. 14-22.

Morse, J. M. \& Field, P. A. (1996). Nursing Research: The Application of Qualitative Approaches ( $2^{\text {nd }}$ Ed.) Standley Thornes, Cheltenham. 
Morse, J. M., Barett, M., Mayan, M., Olson, K., \& Spiers, J. (2002). Verification Strategies for Establishing Reliability and Validity in Qualitative Research. International Journal of Qualitative Methods 1 (2) 119.

Polit, D. F., \& Beck. C. T. (2012). Nursing Research: Generating and Assessing Evidence for Nursing Practice ( $9^{\text {th }}$ Edition). China: Wolters Kluwer Health, Lippincot Williams \& Wilkins. ISBN 978-1-60547-708-4. Rolfe, G. (2006). Validity, Trustworthiness, and Rigour: Quality and the Idea of Qualitative Research. Journal of Advanced Nursing. University of Wales Swansea. United Kingdom: Blackwell Publishing Ltd.

Saldana, J. (2011). Fundamentals of Qualitative Research, Understanding Qualitative Research. New York, USA: Oxford University Press, Inc. ISBN 978-0-19-973795-6.

Salvador, J. (2015). HOPE Beyond the Ageing Lines: the Lived Experiences of Filipino Elderly in Metro Manila, Philippines. International Journal of Human Caring (Vol. 4, Issue 4). USA: Michigan. Innovative Print \& Media Group Inc.

Sandelowski, M. (1993). Rigor or Rigor Mortis: The Problem of Rigor in Qualitative Research Revisited. Advances in Nursing Science 16(2), 1-8.

Sandelowski, M. \& Barroso, J. (2002). Reading Qualitative Studies. International Journal of Qualitative Methods (Vol. 1, Issue 1, Article 5. Retrieved from http://www.ualberta.ca/ ijqm/ on 06 July 2004.

Somekh, B., \& Lewin, C. (2012). Theory and Methods in Research $\left(2^{\text {nd }}\right.$ Edition). New Delhi, India: Sage.

Van Manen, M. (1989). Pedagogical Text as Method: Phenomenological Research as Writing. University of Alberta: Saybrook Review (Vol. 7, No. 2).

Willis, J. (2007). Foundations of Qualitative Research: Interpretive and Critical Approaches. United States of America: Sage Publication, Inc. 